\title{
Reward sensitivity for a palatable food reward peaks during pubertal developmental in rats
}

\section{Chris M. Friemel, Rainer Spanagel and Miriam Schneider*}

Department of Psychopharmacology, Central Institute of Mental Health, Heidelberg University, Mannheim, Germany

\section{Edited by:}

Andrew Holmes,

National Institute of Health, USA

Reviewed by:

Tracy L. Bale,

University of Pennsylvania, USA

Alicia Izquierdo, California State

University, USA

*Correspondence:

Miriam Schneider, Department of Psychopharmacology, Central Institute

of Mental Health (ZI), J5, 68159

Mannheim, Germany.

e-mail:miriam.schneider@zi-

mannheim.de
Puberty is a critical period for the initiation of drug use and abuse. Because early drug use onset often accounts for a more severe progression of addiction, it is of importance to understand the underlying mechanisms and neurodevelopmental changes during puberty that are contributing to enhanced reward processing in teenagers. The present study investigated the progression of reward sensitivity toward a natural food reward over the whole course of adolescence in male rats (postnatal days 30-90) by monitoring consummatory, motivational behavior and neurobiological correlates of reward. Using a limited-free intake paradigm, consumption of sweetened condensed milk (SCM) was measured repeatedly in adolescent and adult rats. Additionally, early- and mid-pubertal animals were tested in Progressive Ratio responding for SCM and c-fos protein expression in reward-associated brain structures was examined after odor conditioning for SCM. We found a transient increase in SCM consumption and motivational incentive for SCM during puberty. This increased reward sensitivity was most pronounced around mid-puberty. The behavioral findings are paralleled by enhanced c-fos staining in reward-related structures revealing an intensified neuronal response after reward-cue presentation, distinctive for pubertal animals. Taken together, these data indicate an increase in reward sensitivity during adolescence accompanied by enhanced responsiveness of reward-associated brain structures to incentive stimuli, and it seems that both is strongly pronounced around mid-puberty. Therefore, higher reward sensitivity during pubertal maturation might contribute to the enhanced vulnerability of teenagers for the initiation of experimental drug use.

Keywords: puberty, reward sensitivity, food reward, c-fos, motivation

\section{INTRODUCTION}

The developmental period of pubertal maturation is a time of dramatic neurobiological and concomitant behavioral changes, including inter alia high risk taking behavior, novelty seeking and impulsivity. High levels of novelty seeking, sensation seeking, and impulsivity have been suggested as strong predictors of drug use (Piazza et al., 1989; Wills et al., 1994; Dalley et al., 2007). It is therefore not surprising that teenagers show higher rates of experimental drug use than adults (Patton et al., 2004; Paus et al., 2008). Evidence from epidemiological studies indicates that the onset of drug use and abuse is most commonly observed during puberty or midadolescence (Patton et al., 2004) and this early substance abuse has been found to predict later adult substance use and dependence (Anthony and Petronis, 1995).

Puberty and adolescence are important developmental periods during which an individual matures from a biologically nonreproductive, infertile child into an adult. Puberty refers exactly to the time period during which sexual maturation is achieved, and is not a unique human developmental period, but can be observed in a variety of different species, including rodents. Rodents display all prominent features of puberty like distinctive hormonal changes, intense peer interactions, increased risk, and novelty seeking (for review see Spear, 2000; Macri et al., 2002). Puberty is initiated by an increased secretion of gonadotropin-releasing hormone $(\mathrm{GnRH})$ and numerous neurodevelopmental alterations are taking place during this period, such as maturational processes in cortical and limbic regions, which are characterized by both progressive and regressive changes, e.g., myelination and synaptic pruning (Spear, 2000; De Bellis et al., 2001; Powell, 2006), indicating that connections and communication between subcortical and cortical regions are in a highly transitional state. In contrast to puberty, adolescence refers to the gradual behavioral and cognitive transition from childhood to adulthood, including the pubertal period, and the boundaries of adolescence are less precisely defined (Schneider, 2008). However, gonadal and neuronal alterations in puberty and adolescent behavioral maturations are intimately linked in timing through multiple and complex interactions between the nervous system and gonadal steroid hormones (Sisk and Foster, 2004).

All these ongoing neurobiological alterations are important for the maturation of adult-like behavioral characteristics, but at the same time render the teenage brain vulnerable for all kind of disturbances.

Aside from severe long-lasting consequences on brain functionality and cognitive impairments caused by pubertal drug exposure (Schneider, 2008; Spanagel, 2009), it is of major importance to understand the underlying neurobiological mechanisms that render adolescents particularly susceptible to drug effects, since this knowledge might help to create better prevention strategies for human teenagers (Spear and Varlinskaya, 2010). 
It is well known from human and animal studies that reward sensitivity is altered during adolescence (Spear, 2000; Galvan et al., 2006). Adolescents show enhanced consummatory behavior for appetitive reinforcers such as food (hyperphagia) and demonstrate also higher reward seeking behavior (for review see Spear, 2000). Most importantly, this change in reward sensitivity may extend to alcohol and drugs of abuse as well, since it is thought that incentive perception of natural and drug rewards share common neurobiological pathways (Spanagel and Weiss, 1999; Kelley and Berridge, 2002) and might therefore underlie the enhanced susceptibility toward drug abuse. However, it is not clear if the increase in seeking and consumption of appetitive rewards during development is related to an increase or a decrease in the incentive value of rewarding stimuli. On one hand, adolescents might seek out natural and drug rewards in order to compensate for a deficit in reward perception. In contrast, reward seeking may also be driven by an increase in the hedonic value (Spear, 2000; Wilmouth and Spear, 2009). Although, some recent studies are supporting the later assumption and indicate that reward perception is higher in adolescents (Vaidya et al., 2004; Wilmouth and Spear, 2009), there is so far no neurobiological proof from animal research for this theory and in addition, the exact timing and duration of these alterations in reward sensitivity have not been investigated so far.

With the present study we were therefore aiming to characterize the progression of reward sensitivity from early-puberty until adulthood in rats. Using sweetened condensed milk (SCM) as a natural reward (Schneider and Spanagel, 2008; Schneider et al., 2010), we measured consummatory as well as motivational aspects of reward perception during the whole time course of adolescence including the pubertal period. In addition to the behavioral data, appetitive cue-induced neuronal activity was measured in reward-relevant brain regions in early-, mid-pubertal and adult male rats by c-fos protein expression revealing neurobiological correlates of reward processing.

\section{MATERIALS AND METHODS SUBJECTS}

A total of 95 naive male Wistar Han rats (Harlan Laboratories, AN Venray, Netherlands) were used for the experiments. Juvenile rats had an age of postnatal day (pd) 28 upon arrival. All animals were kept under temperature and light $(12 \mathrm{~h} / 12 \mathrm{~h}$ dark-light cycle, lights on at 7:00 AM) controlled conditions. The animals were housed in groups of five or six in standard macrolon cages (Eurostandard type IV) with lab chow and tap water available ad libitum. To examine the developmental status of pubertal onset, rats were checked daily for complete balano-preputal separation (BPS) as described by Korenbrot et al. (1977) starting from age pd 38. The daily check of the development of BPS revealed a mean puberty onset at an age of pd $42 \pm 1.8 \mathrm{SD}$ in our male Wistar rats with $88 \%$ of all animals starting puberty until pd 43.

All experiments were conducted in accordance with the ethical guidelines for the care and use of laboratory animals, and were approved by the local animal care committee (Regierungspraesidium Karlsruhe, Germany).

\section{EXPERIMENTAL DESIGN}

Different groups of animals were used for all three experiments: SCM intake (1), progressive ratio (PR) testing (2) and c-fos stimulation by appetitive odor conditioning (3), to assess the development of different behavioral and neurobiological aspects of reward sensitivity throughout adolescence, in particular during the pubertal period, until adulthood. Puberty in male rats reaches from around pd 40 to pd 60 (Schneider, 2008). In contrast to puberty, the exact timing of adolescence is rather difficult to define in laboratory animals. Per definition, adolescence covers the complete time span from childhood to adulthood, including the pubertal period (Sisk and Foster, 2004). Therefore, adolescence begins in the juvenile period directly after weaning (around pd 22), extending to sexual maturity by the end of puberty, and continuing into early adulthood ( $\sim$ pd 70/80 in male rats).

For all behavioral experiments SCM (Nestle AG, Frankfurt, Germany; diluted 1:3 with tap water) was used as the rewarding stimulus. Two days prior the first experimental testing rats were habituated to SCM over night in their home cages. (1) SCM intake was tested repeatedly in a group $(n=12)$ of adolescent animals at pd 30,40,50,60, 70, 80 and 90 to gain consummatory data covering the whole period from pre-puberty to adulthood. Additionally, the progress of weight gain was continuously measured in these rats. Body weights were recorded every tenth day and percentage weight gain in relation to previous body weight was calculated by using the formula $\{$ [weight gain $(\mathrm{t} 1-\mathrm{t} 2) / \mathrm{body}$ weight $(\mathrm{t} 1)] \times 100\}$. Additionally, a second group of adult animals $(n=12)$ was repeatedly tested for SCM consumption on pd 100, 110,120 and 130 to control for the effects of repeated testing. (2) For the PR experiment three groups of animals were tested: earlypubertal rats aged pd 40 at the testing day $(n=15)$, mid-pubertal rats aged pd 50 at the testing day $(n=15)$ and adult animals aged 90 days $(n=11)$. (3) Finally, cue-induced c-fos stimulation was done in three different age groups, with half of the animals trained for odor-association and the other half sham-trained. Odor exposure took place on either pd $40(n=10)$, pd $50(n=10)$, or pd $90(n=10)$.

\section{BEHAVIORAL TESTING Limited-access SCM intake}

Consummatory behavior for a natural reward was measured using a time-limited-free consumption paradigm (Schneider et al., 2010). Animals were separated in single macrolon cages (Eurostandard type III) and allowed to habituate to the new environment for 5 min. A bottle of SCM solution was than introduced into the cage and animals were allowed to drink ad libitum within a period of $15 \mathrm{~min}$. Afterward, the animals were returned into their home cages. The amount of SCM consumed ( $\mathrm{ml}$ ) during the 15-min test period was recorded for each animal and calculated per kilogram bodyweight ( $\mathrm{ml} / \mathrm{kg} \mathrm{BW})$.

\section{Progressive ratio testing}

Progressive ratio testing and training were conducted in a standard operant chamber $(30.5 \mathrm{~cm} \times 24.1 \mathrm{~cm} \times 21.0 \mathrm{~cm}$, Med Associates Inc., St. Albans, USA). On the first day, animals were habituated to the skinner boxes and received free access to SCM in the chamber (shaping). For the following 4 days rats were trained to lever press for $90 \mu \mathrm{l} \mathrm{SCM}$ in 30-min training sessions on a continuous reinforcement schedule (CRF) until they reached stable baseline responding (at least 60 lever presses per session). One day later a 30-min PR test session was conducted with a ratio requirement for successive 
rewards increasing by a progression of 2 (i.e., 2, 4, 6, 8, ...). Total lever presses, the highest completed ratio (HCR) and the inactivity ratio (IR), defined as the initial break in responding for more than $2 \mathrm{~min}$, were recorded with the IR being set as break point in progressive ratio performance. Additionally, the total amount of SCM received and consumed during PR testing was measured and calculated per bodyweight as (ml/kg BW).

\section{Appetitive odor-conditioning for c-fos stimulation}

In order to elicit c-fos activity by the presentation of an appetitive cue, rats were first trained to associate an orange odor cue with SCM. Rats were trained in 90-min sessions for five consecutive days as described before (Schneider and Spanagel, 2008). For each training session rats were separated in single macrolon cages (Eurostandard type III) and experienced three odor-reward presentations at random time points after a habituation period of $10 \mathrm{~min}$ to the novel environment. Odor-reward presentations involved the introduction of the odor, followed immediately by the presentation of a bottle containing SCM. The odor (orange, $15 \mu \mathrm{l}$ ) was supplied in a small Petri dish containing a piece of filter paper that was placed in the middle of the wire lid, $2 \mathrm{~cm}$ beneath the aperture of the drinking bottle. After free access to the reward for $5 \mathrm{~min}$ the odor and the SCM were removed. Training sessions were randomly conducted during the light phase. In addition to this training group, a second group of animals was sham-trained as controls. These rats received for 5 days at randomized time points within the light phase 15-min access to the SCM in single cages and were additionally exposed to the orange odor in an explicitly non-associative manner $4 \mathrm{~h}$ before or after SCM presentation (Schneider and Spanagel, 2008; Schneider et al., 2010).

To measure c-fos protein expression elicited by the conditioned odor cue in reward-relevant brain regions, rats were exposed at the sixth day only to the orange odor $(15 \mu \mathrm{l})$ for $15 \mathrm{~min}$. Subsequently, rats were brought for $90 \mathrm{~min}$ to a darkened and quiet resting room, to which they had been habituated daily for three consecutive days, to allow optimal protein translation. Animals were than sacrificed and brains were removed, quickly frozen in methylbutan for $90 \mathrm{~s}$ and stored at $-80^{\circ} \mathrm{C}$ until further processing.

\section{IMMUNOHISTOCHEMISTRY FOR C-FOS}

Frozen brains were sliced in $10-\mu \mathrm{m}$ coronal sections on a Cryostat and immediately thaw-mounted onto Superfrost glass slides. For the present study the nucleus accumbens core (NACc) and shell (NACs), as well as the dorsal striatum (dStr), were chosen for regions of interest. After fixing the brain sections with 4\% PFA they were washed with $1 \times$ PBS and endogenous peroxidases were quenched with $0.3 \% \mathrm{H}_{2} \mathrm{O}_{2}$ in $1 \times$ PBS. Slides were washed in $1 \times$ PBS and $1 \times$ PBS/Tween-20 and blocked with goat serum. To improve c-fos signaling separate 15 min blockings for Avidin and Biotin (VectorLab, Burlingame, CA, USA) were conducted. C-fos rabbit polyclonal antibody (Abcam, Cambridge, UK) was diluted 1:100 in 1× PBS and sections were incubated for $30 \mathrm{~min}$ at $37^{\circ} \mathrm{C}$. The second antibody was a biotinylated goat-anti-rabbit IGg (VectorLab, Burlingame, CA, USA) diluted 1:200 in 1× PBS and goat serum and was incubated for $30 \mathrm{~min}$ at room temperature. The Vectastain Elite ABC Kit (VectorLab, Burlingame, CA, USA) was applied to the sections for $30 \mathrm{~min}$ at room temperature before DAB staining. Staining was stopped by rinsing the slides with aqua bidest. Subsequently brain sections were dehydrated by graded ethanol bathes and cleared by Xylol before mounted in Eukitt. Images were captured using Axioskop 2 Microscope (Zeiss, Jena, Germany) with a $20 \times$ air objective (NA 0.5) and an Olympus ColorView 3 camera. At a section plane level of +2 to +1.6 mm from Bregma, according to Paxinos and Watson (1998) rat brain atlas, a $250 \mu \mathrm{m} \times 250 \mu \mathrm{m}$ was randomly positioned into the sampled areas and all c-fos labeled cells in this section were counted using ImageJ.

\section{STATISTICAL ANALYSIS}

Age-dependent effects on SCM intake in adolescent and adult rats and the percentage gain of body weight during adolescence were evaluated using repeated measurement analysis of variance (ANOVA) with Greenhouse-Geisser correction. To precisely calculate differences of pubertal and adult SCM consumption a Dunett test (with pd 90 assigned as control group) and a $t$-test had been conducted. For PR performance each parameter was separately analyzed using one-way ANOVA. For c-fos protein expression data a multivariate analysis of variance (MANOVA) was applied with two main factors (age and training) and three dependent variables (c-fos counts in NACc, NACs, and dStr). The Fisher LSD test for pair-wise comparisons was used by default for all post hoc analysis. The alpha value was set to 0.05 for all tests. All statistical calculations were performed in SPSS.

\section{RESULTS \\ LIMITED-ACCESS SCM INTAKE}

Intake of SCM varied significantly over the complete testing phase from pd 30 to $\mathrm{pd} 90$ (repeated measurement ANOVA, $F_{2.9,31.9}=17.48$ $p \leq 0.001$ ) (Figure 1A). A remarkable boost in SCM consumption at pd 50 differed significantly from measures in younger $(p=0.03 / 0.047$ for pd 30/pd 40, respectively) and older rats $(p \leq 0.001)$ as revealed by post hoc analysis with Fisher LSD test. After this peak intake levels descended to an adult niveau that stabilized around pd 80 and pd 90. While SCM consumption at pd 60 was still significantly increased in comparison to pd $90(p=0.004)$ drinking data at pd 70, pd 80 and pd $90(p>0.05)$ did not differ statistically significant anymore. In comparison to adult animals (pd 90) the SCM intake of prepubertal and pubertal rats ( $\mathrm{pd} 30-\mathrm{pd} 60$ ) was significantly elevated (Dunett test, $p \leq 0.009$ ). Testing the group of pubertal (pd 40-pd 60) against adult ( $\mathrm{pd} 90$ ) rats revealed significantly higher intake of SCM during puberty ( $t$-test, $t_{46}=4.69 p \leq 0.001$ ) (Figure 1C). Repeated measurement of SCM consumption in adult rats revealed no retest effect (repeated measurement ANOVA, $F_{1.7,18.9}=0.41 p=0.64$ ) (Figure 1B). Monitoring the body weight and the percentage weight gain in the course of adolescence showed the highest weight gain between pd 30 and pd 50 with more than 60 g mean increment in 10 days followed by attenuated weight gains that pursue a logarithmic function. Calculating the percentage gain in relation to body weight revealed the peak increment between pd 30 and pd 40 with a mean growth of $75 \%$ and a subsequent drop to $44 \%$ (pd 40-50), $19 \%$ (pd 50-60) and so on (Figure 2). Statistically the percentage body weight gain differed highly significant during adolescence (repeated measurement ANOVA, $\left.F_{1.9,21.8}=52.45 p \leq 0.001\right)$ with significant higher weight gains from pd 30 to pd 60 ( $p \leq 0.001)$. 


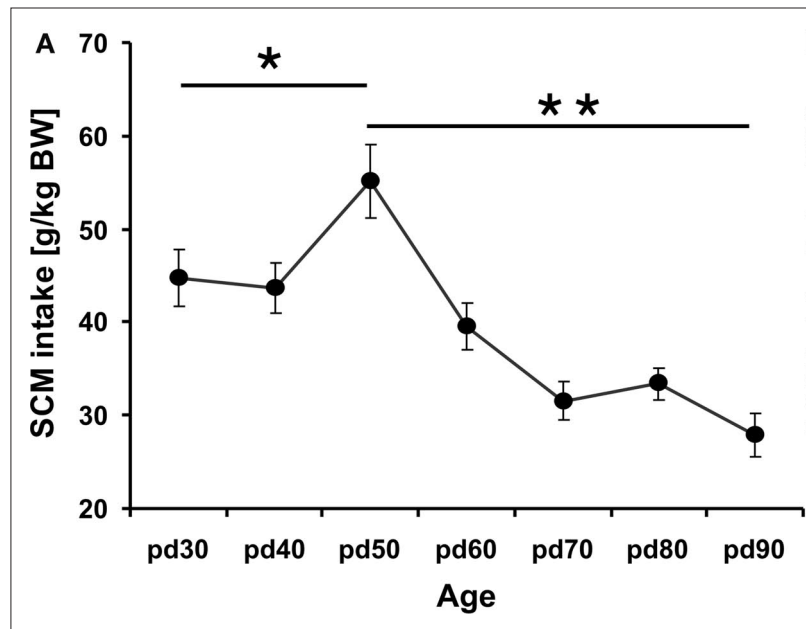

FIGURE 1 | Mean SCM intake relative to body weight in adolescent rats between age pd 30 and pd $90(A)$ and in adult rats (starting at pd 100) (B). SCM intake was increased during early and mid-adolescence (pd 30-60) compared to young adulthood (pd 70-90). A peak was observed on pd 50 that differed significantly from all other testing days. No statistical differences were
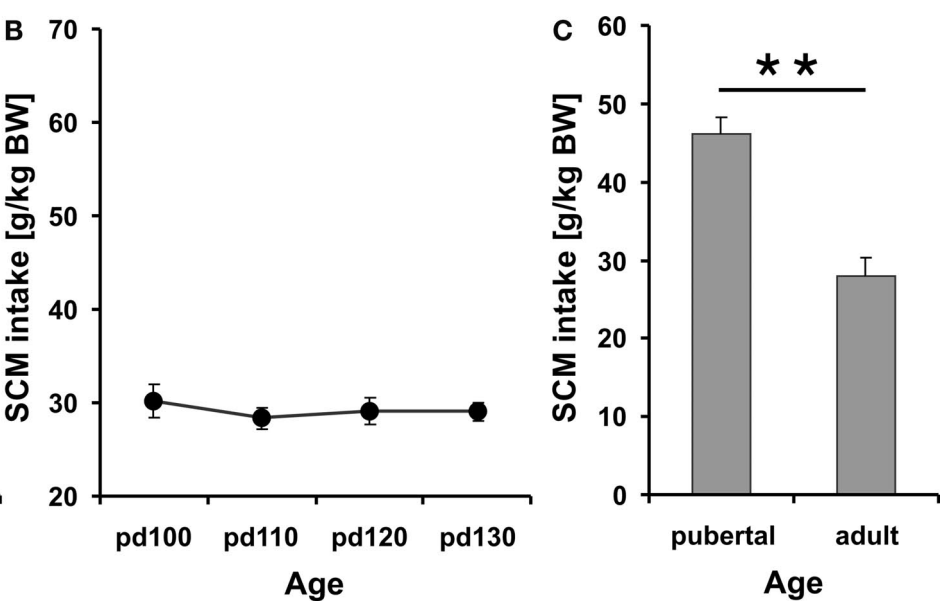

observed after repeated testing in adult rats (B). In addition, the average SCM consumption of pubertal rats (PND 40-60) differed significantly from adult animals on pd 90 (C). Asterisks $\left({ }^{*}\right)$ and $\left({ }^{*}\right)$ indicate significant differences at $p$-levels of $p \leq 0.05$ and $p \leq 0.01$, respectively. Data are expressed as mean \pm SEM.

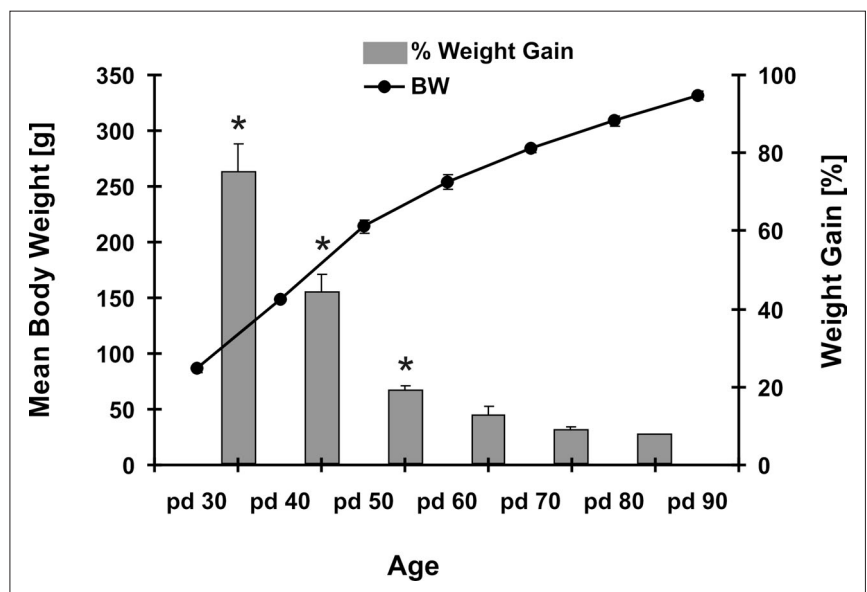

FIGURE 2 | Mean body weight and percentage weight gain during adolescence. Points indicate mean body weight of rats between an age of PND 30 to PND 90 (left scale). Bars indicate the percentaged weight gain in relation to previous body weight (right scale). The highest weight gain was observed during pubertal development, with a peak just shortly before puberty onset from pd 30 to 40 . Asterisks $\left({ }^{*}\right)$ indicate significant differences at $p$-levels of $p \leq 0.05$. Data are expressed as mean \pm SEM.

\section{PROGRESSIVE RATIO}

During the PR test session mid-pubertal pd 50 rats pressed significantly more often the lever to obtain SCM compared to younger and adult rats (ANOVA $F_{2,38}=4.23 p=0.02$ Fisher LSD $p=0.05 / 0.008$ for pd 40/adult, respectively) (Figure 3A). Accordingly, the HCR was significantly higher for pd 50 rats (ANOVA $F_{2,38}=4.78 p=0.01$ Fisher LSD $p=0.05 / 0.005$ for pd 40/adult, respectively) (Figure 3B). Total lever presses and HCR of early-pubertal and adult rats did not differ significantly $(p>0.05)$. No difference was found in the break point for all three age groups (ANOVA $F_{2,38}=1.37 p=0.27$ ) (Figure $3 \mathrm{C}$ ). Comparing the consumption of SCM corrected for body weight within PR testing revealed highly significant differences between all groups (ANOVA $F_{2,38}=53,68 p \leq 0.001$ ). While pd 40 old rats had the highest intake, both pubertal groups differed significantly from adult animals ( $p \leq 0.001)$ (values: pd 40: $5.35 \pm 0.27$; pd 50: $4.18 \pm 0.17$; pd 90: $2.17 \pm 0.13$ all values are in $\mathrm{ml} / \mathrm{kg}$ $\mathrm{BW} \pm \mathrm{SE})$.

\section{C-FOS PROTEIN EXPRESSION}

One animal of the adult/odor-conditioned group needed to be excluded from further analysis due to staining inconsistencies. The conditioned odor stimulus significantly activated c-fos protein expression in all age groups in both NACc and NACs and in the dStr in rats conditioned for SCM in comparison to sham controls (MANOVA, Wilk's $\lambda=0.365 F_{3,25}=14.48 p<0.0001$ ) (Figures 4 and 5). In sham-trained rats $c$-fos expression levels of the examined brain regions did not show age-related variations. In contrast, in conditioned animals the number of c-fos active cells differed clearly with age in NACc and dStr. Animals tested on pd 40 and pd 50 had significantly more c-fos activated cells in the NACc compared to adult rats (Fisher LSD $p=0.03 / p=0.01$, respectively). Similarly, in the $\mathrm{d}$ Str the highest number of $\mathrm{c}$-fos labeled cells was observed at pd 50 followed by pd 40 with both levels being significantly increased compared to adult rats (Fisher LSD $p=0.028 / p=0.048$, respectively). Although, the number of $c$-fos expressing cells in NACs was slightly higher in trained pubertal rats than in adult ones, this difference did not reach statistical significance (Fisher LSD $p>0.05$ ).

\section{DISCUSSION}

With the present study we could show a transient up-regulation of consummatory and motivational behavior for a natural food reward during pubertal development in male Wistar rats. We observed a strong increase in SCM intake that was accompanied by enhanced motivational incentive responding for this reward during pubertal development, which both declined 

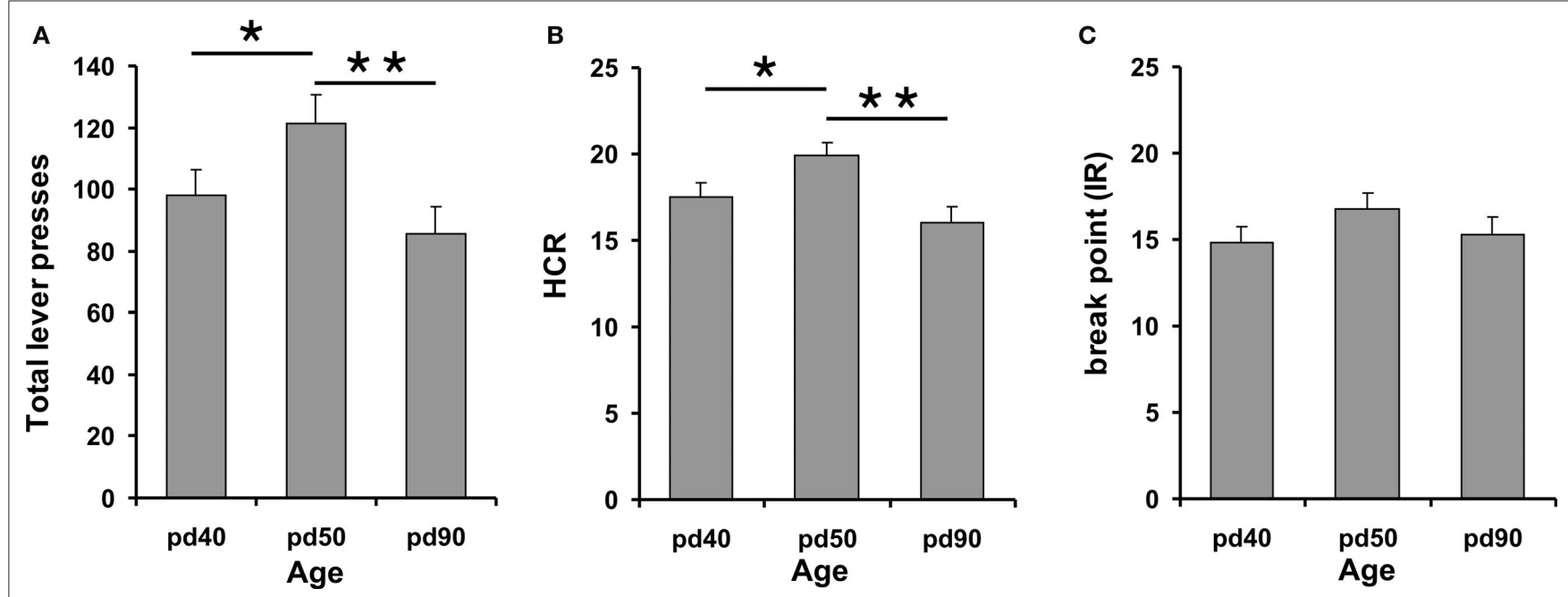

FIGURE 3 | Progressive Ratio performance of early- (pd 40), mid-pubertal (pd 50) and adult rats (pd 90). The figure depicts total lever presses (A), HCR significantly from early-pubertal and adult animals for total lever presses and (B) and the break point (IR) (C). Performance of mid-pubertal rats differed

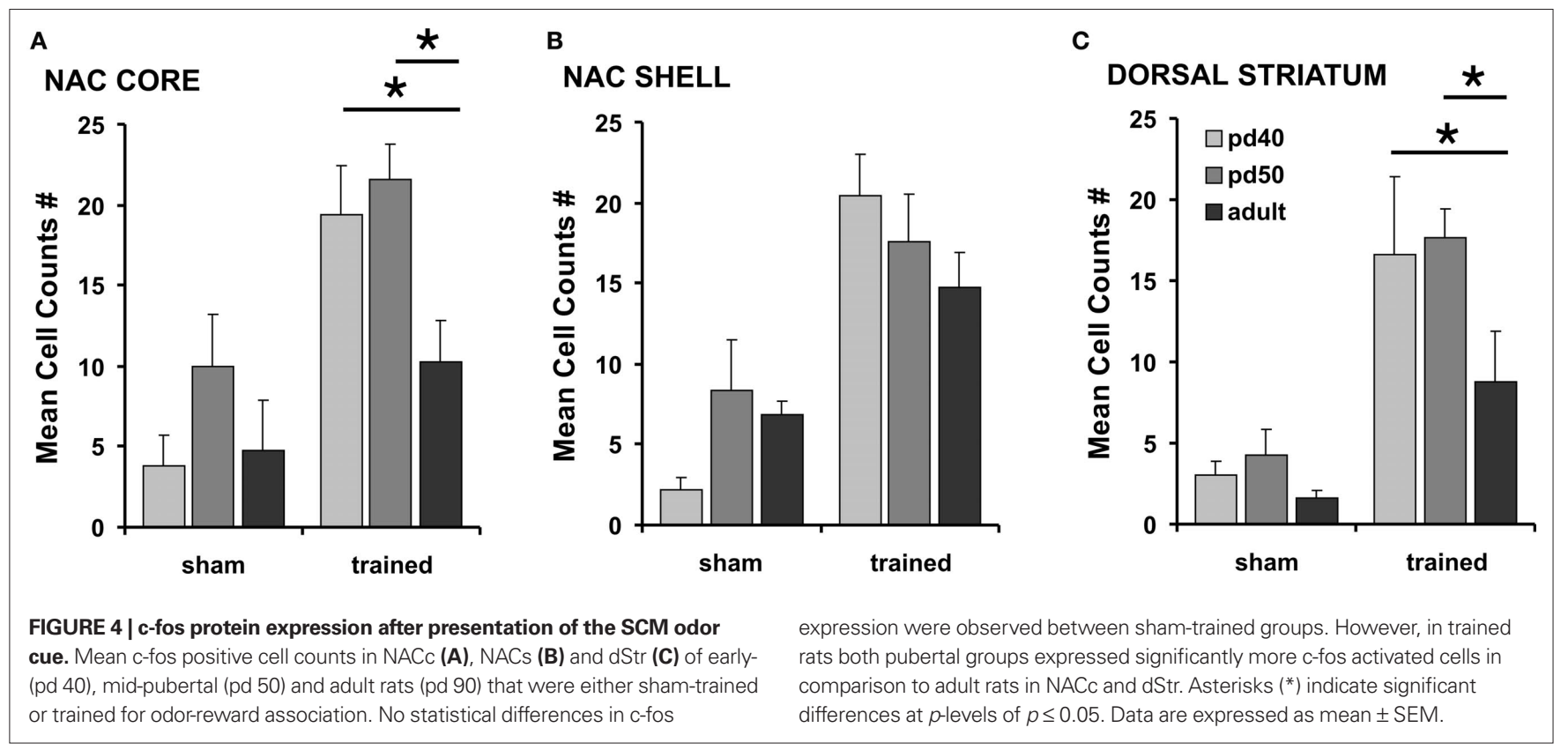

at adulthood. Comparing neuronal activity in reward-related structures between early-, mid-pubertal and adult rats after reward-associated stimulus presentation revealed significant higher c-fos protein expression in NACc and in dStr for pubertal odor-conditioned rats while c-fos expression did not differ at any age in sham-trained rats. Even though early-pubertal rats had significant higher expression of c-fos protein compared to adults, this effect was more pronounced in mid-pubertal rats. The period of strongest sensitivity toward natural rewards was found at early mid-puberty characterized by increased reward consumption, enhanced motivation and effort to obtain rewards and a stronger induction of c-fos protein in reward-related structures after reward-associated cue presentation.

\section{LIMITED-FREE SCM INTAKE}

The repeated measurement of SCM intake in a limited-access paradigm from pd 30 (pre-puberty) to pd 90 (adulthood) revealed a significant higher intake, relative to body weight, during the pubertal period compared to adulthood. This high pubertal intake of a palatable liquid is in line with data reported for $1 \%$ sucrose solution in early adolescent male Sprague-Dawley rats tested between pd 28 and pd 42 (Wilmouth and Spear, 2009). In this study the 24-h intake of water and 1\% sucrose was measured in a 2-bottle-choice test over 14 days. Adolescent rats drank significantly more sucrose than adults and also more than water, whereas in adult rats intake of water and sucrose did not differ. The longitudinal design of our study allowed a systematic monitoring of the progression of 


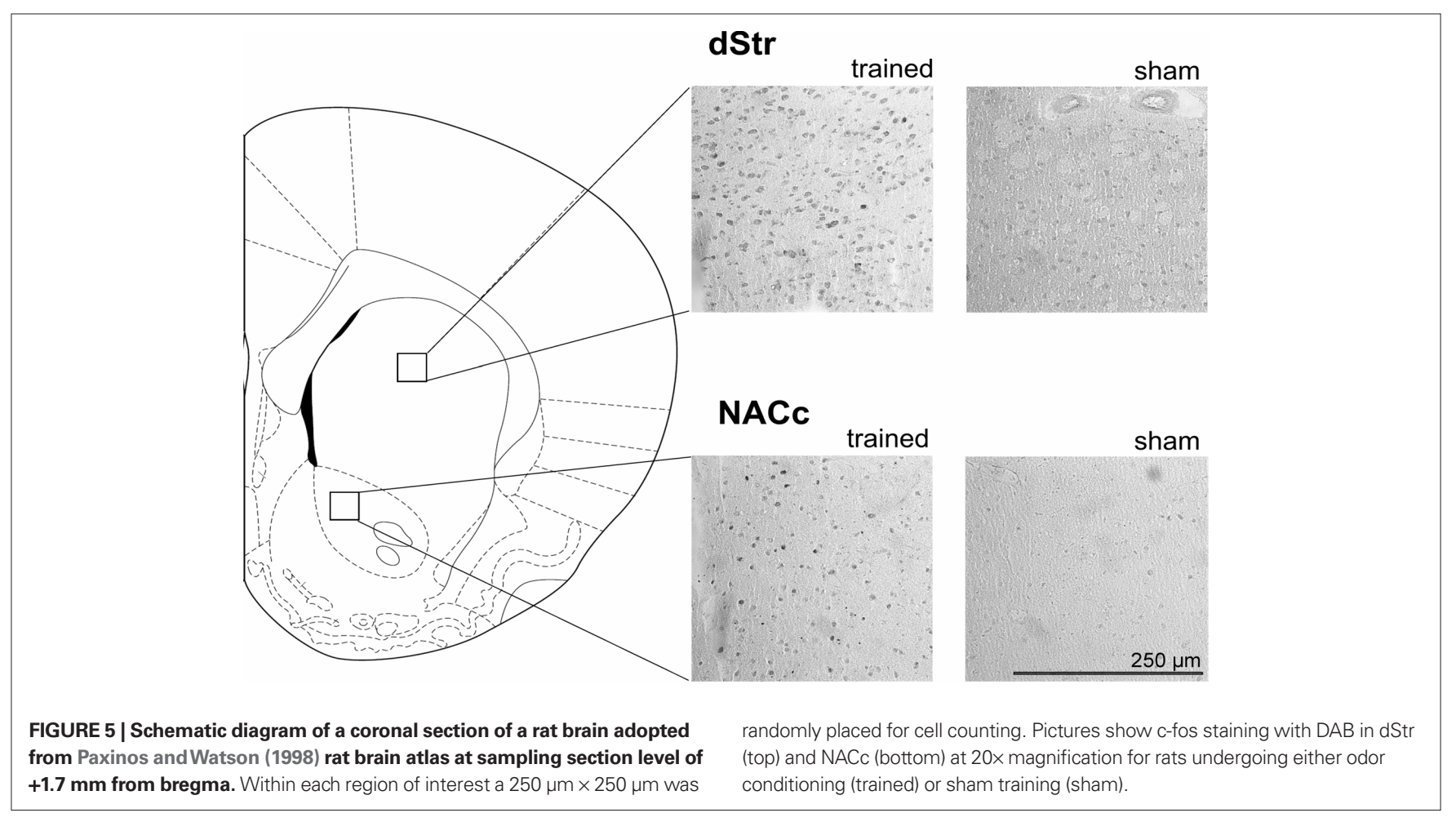

adolescent natural reward consumption beyond pd 42. The peak in SCM consumption was seen at pd 50 followed by a steep decline until pd 70 and a further decrease to a stable adult consumption level that was reached by pd 90 . By using the criterion of BPS as clearly definable marker of pubertal onset the time window of puberty in male rats is considered roughly between pd 40 and pd 60 (Schneider, 2008). From the present data it therefore appears that during adolescence the restricted period of pubertal developmental is the most sensitive time window for the elevation of reward intake.

Consumption of natural rewards is mainly driven by palatability and the homeostatic state of the organism. A major point of consideration for consummatory tests during development is the adolescent hyperphagia, with humans and rats exhibiting higher caloric intake relative to their body weight than at any other time in the life span (Nance, 1983; Post and Kemper, 1993). To minimize an interference of varied caloric demands with SCM consumption rats were permanently fed ad libitum. Higher consumption of SCM during the short experimental access of 15 min might therefore reflect a more increased liking of the reward instead of compensating caloric demands in pubertal rats. This is further supported by our findings regarding weight gains during puberty, since the increase in body weight followed a logarithmic function with the highest growth spurt observed between pd 30 and 40 . The caloric demand should be therefore highest in this specific time span. Accordingly, a potential influence of heightened caloric demand on SCM intake would also be expected to be strongest between pd 30 and pd 40. The peak in SCM consumption observed at pd 50 can therefore be considered as being less affected by caloric demands and mostly influenced by enhanced sensitivity toward the rewarding properties of SCM. Although, no direct correlation could be observed between the peak in consumption and the highest growth spurt with beginning of puberty, a potential preference for high caloric foods during periods of dynamic growth like adolescence might be important from an evolutionary perspective and therefore an influence of caloric demands can not be excluded completely. However, it has been shown that increased reward sensitivity during adolescence is not restricted to food rewards but can been seen for other naturals rewards and drug rewards as well, independent from caloric contents (Spear, 2000; Brenhouse and Andersen, 2008).

Similar results supporting the assumption that the caloric content is not primarily important for the consumption of palatable food have been reported before by Gilbert and Sherman (1970). They found a comparable liquid intake of sucrose and non-caloric saccharin solutions in food-deprived rats, indicating that the sweet taste has a stronger impact on consumption compared to the nutritional value of a palatable fluid. Additionally, consistent with our present findings higher preference for sweet tastants was observed during puberty in both, rodents and humans (Desor and Beauchamp, 1987; Vaidya et al., 2004), indicating a transient increase in sensitivity toward the appetitive properties of food rewards during development. Further evidence for a changed hedonic sensitivity toward appetitive and aversive food stimuli during adolescence was provided by Wilmouth and Spear (2009). Using the taste reactivity paradigm which is thought to directly measure the hedonic value of orally presented stimuli (Grill and Norgren, 1978), they measured stereotypic positive and negative behaviors of adolescent (pd 30-34) and adult rats (pd 72-73) in response to oral infusions of differently concentrated sucrose solutions or a quinine containing liquid. Adolescent rats exhibited significantly more positive responses than adult animals during infusion of $10 \%$ sucrose and increased this responding already at a sucrose 
concentration of $3.4 \%$ at which the response pattern of adult rats did not differ from water condition. Contrary, infusion of quinine elicited only in adult rats significant more negative responses in comparison to water.

Taken together, this data indicate that the sensitivity toward positive hedonic aspects of rewarding stimuli is transiently enhanced while for negatively valued stimuli it is attenuated in adolescent rats and these findings are consistent with our present observation of enhanced SCM intake during puberty.

In our study repeated testing of SCM intake in adult rats showed constant drinking volumes without any re-test effects. These data demonstrate that repeated testing for SCM intake does not produce habituation nor sensitization for this food reward and therefore indicates that the characteristic course of SCM intake during adolescence is not related to any confounding factors.

\section{PROGRESSIVE RATIO}

To evaluate potential changes in motivation for SCM during earlypuberty, mid-puberty and adulthood, animals in the according age span were tested in a PR paradigm. Since an increasing number of lever presses for each subsequent reinforcer is required in this task, PR performance has commonly been used as an index of motivation and the effort a subject is willing to exert in order to obtain a reinforcer (Hodos and Kalman, 1963; Stafford et al., 1998; Sanchis-Segura and Spanagel, 2006). The performance in PR for SCM revealed significant higher total lever presses and HCR for mid-pubertal pd 50 rats compared to pd 40 pubertal and adult animals. Pubertal rats did not differ in performance from adult rats. However, when taking the higher physical effort of lever pressing for young rats into account compared to outgrown rats (pd 40 and adult rats had mean body weights of $147 \mathrm{~g}$ and $331 \mathrm{~g}$, respectively), it is remarkable that early-pubertal rats performed equivalently in total lever presses and HCR to adult animals.

No differences between the different groups were found regarding the occurrence of the break point (defined as inactivity over a period of $2 \mathrm{~min}$ ). The occurrence of the break point is not only influenced by motivational aspects, but frustration induced by unexpected reward-omission might also contribute to this first break in perseveration. In this context we could show before in a previous study that although total lever presses and HCR during a 30-min session were highly reduced by the opioid receptor antagonist naloxone, this treatment did not affect break points for SCM, indicating a differential neuronal regulation of these measures (Schneider et al., 2010). Therefore, the data obtained in our present study are indicating that age is not influencing the reaction to frustrative events, however, after the occurrence of this first break in responding mid-pubertal rats continued to invest more effort for the palatable food reward resulting in a total higher number of lever presses and HCR compared to early-pubertal and adult animals. A higher effort invested by pubertal rats for SCM could be mediated by a potential increase of motivational incentive for SCM during this age. Earlier reports already indicate enhanced motivation for other natural rewards like social play or novelty in adolescent rats (for review Doremus-Fitzwater et al., 2010). In these studies a conditioned place preference (CPP) paradigm had been used as an indirect measure of motivation to approach a compartment that was previously paired with a social partner or novel objects, respectively (Douglas et al., 2003, 2004). In both cases, the ratio of time spent in the paired compartment to the non-paired one was higher for male adolescent rats (pd 38) compared to adults. However, pubertal ( $\mathrm{pd} 40$ ) and adult rats, trained to lever press for saccharin, did not differ in the number of lever presses when tested under fixed or PR schedules (Shram et al., 2008). Despite similar operant responses, a significant higher saccharin consumption during the test sessions was observed in adolescent rats when the saccharin reinforcements received were adjusted for the animals' body weight. This difference was most pronounced under the highest fixed ratio (FR 5) schedule and with escalating saccharin concentrations. When calculating the amount of reinforcers (SCM) obtained (adjusted for body weight) in our PR test, we detected a similar result. The highest amount of SCM in relation to bodyweight was obtained by early-pubertal and mid-pubertal rats in comparison to adult animals. The adjusted consumption during the PR test strongly supports the interpretation of enhanced motivation for natural rewards during adolescence but also points to a careful performance analysis of instrumental behavior in developmental studies. The influence of partly substantial differences in body weight, physical effort or intake capacity of tested animals needs to be considered in the interpretation of operant performance and age comparison.

Behavior measured in PR is influenced by multiple variables such as motivational incentive and hedonic aspects of the reinforcer or the internal state of the subject. Comparing activation and neurotransmitter release in neuronal substrates related with incentive and motivational processing in adolescent and adult humans and animals revealed marked maturational changes in subcortical and prefrontal brain regions and their neurocircuitries (Chambers et al., 2003; Crews et al., 2007; Casey et al., 2008; Ernst et al., 2009; Geier and Luna, 2009). During adolescence an enhanced monoaminergic activation of the fronto-striatal system (Nakano and Mizuno, 1996; Andersen and Teicher, 2000; Tarazi and Baldessarini, 2000; Haycock et al., 2003) is associated with higher levels of impulsivity and increased responsiveness to rewards and drugs (Chambers et al., 2003; Kuhn et al., 2009; Doremus-Fitzwater et al., 2010). Likewise, considerable developmental changes have been reported for the two neuropeptide systems proposed to mediate the hedonic aspects of reward, namely the opioid and the endocannabinoid system (Solinas and Goldberg, 2005; Solinas et al., 2007; Ellgren et al., 2008). A transient up-regulation of the endocannabinoid system during adolescence is indicated by enhanced radioligand binding on cannabinoid receptor 1 (CB1) in limbic structures (Rodriguez de Fonseca et al., 1993) or increased anandamide expression at pubertal onset (pd 38) in multiple brain regions like, for example, NAC and caudate putamen (Ellgren et al., 2008) or hypothalamus (Wenger et al., 2002; for detailed review see Schneider, 2008). Although, stable levels of Met-enkephalin and $\mu$ opiod receptors $(\mu \mathrm{OR})$ were reported during adolescence (Ellgren et al., 2008), higher stimulatory effects of the $\mu \mathrm{OR}$ agonist DAMGO on social facilitation were found during early adolescence (Varlinskaya and Spear, 2008).

Integrating the neurodevelopmental changes accompanying adolescence strongly indicates a higher sensitivity toward hedonic and motivational aspects of natural rewards as well as of drugs of abuse during this developmental phase due to an enhanced 
neurobiological response toward rewarding stimuli. The higher effort and consumption of SCM observed for pubertal rats in PR further supports this assumption. In accordance with our consummatory data for SCM, the motivational effect was more pronounced in mid-pubertal rats.

\section{C-FOS EXPRESSION}

The expression of $\mathrm{c}$-fos protein levels, the translated product of the immediate early gene c-fos, is generally considered a marker of neuronal activation in specific brain regions in response to multiple stimuli (Hughes and Dragunow, 1995). In the present study c-fos protein levels were measured in dorsal and ventral striatum after presentation of a SCM-associated odor cue in rats of pubertal (pd 40 and 50) and adult age (pd 90).

The access to palatable food rewards, such as sucrose solutions, has been shown to robustly induce c-fos expression in different brain areas in rats (e.g., Streefland et al., 1996; Grippo et al., 2004; Norgren et al., 2006; Mungarndee et al., 2008). Even the presentation of a cue that had been previously paired with a reward can activate c-fos expression (Miller and Marshall, 2005; Zhang et al., 2005). In our present study, we found that c-fos expression after odor-cue presentation was always higher in groups that underwent odor-reward association training, irrespective of age, compared to sham-trained control groups, indicating a specific increase in neuronal activity in response to the conditioned odor. In addition, we observed a significant higher c-fos expression in the dStr and in NACc in both pubertal groups compared to adult rats. Even though more c-fos labeled cells were detected in all pubertal rats the increase was more pronounced in pd 50 animals. In the NACs no significant differences between pubertal and adult animals could be found.

Although, c-fos activity in reward-associated brain structures is considered to reflect only a correlative measure of reward perception and reward processing, a direct influence of reward magnitude on the induction of c-fos expression was demonstrated after intracranial self-stimulation in rats (Panagis et al., 1997). Our findings are indicating enhanced neuronal activity of reward-related brain structures after presentation of the reward-associated cue in pubertal rats and are therefore supporting the hypothesis that rewarding stimuli have a higher incentive during adolescence and not the opposite. This finding is consistent with other studies in humans and rodents (Laviola et al., 2003; Ernst et al., 2005; Galvan et al., 2006; Mungarndee et al., 2008; Kringelbach and Berridge, 2009). For example, Ernst et al. (2005) monitored brain activity of adolescent and adult human subjects during anticipation, gain or omission of monetary rewards with fMRI. Teenagers showed higher activity in NAC when gaining a reward compared to adults.

The pronounced c-fos protein expression in dStr observed in our study is consistent with other studies reporting enhanced and sustained activation in this region after reward presentation (Delgado et al., 2003). Similarly, fMRI studies typically report increases in blood oxygenation level dependent (BOLD) responses in the dStr during anticipation of either primary (O'Doherty et al., 2002) or secondary (Knutson et al., 2001) rewards. The dStr is also considered as a major component of the brain's motivational and reward system (Wise, 2009). This dopaminergic subsystem is known to be critically involved in motivational and reward-associated processes (Wise, 2004) and underlies maturational changes during puberty (Spear, 2000; Andersen, 2002). This enhanced susceptibility and reactivity of reward-related brain structures toward rewards during adolescence is reflected by our present finding of significant higher c-fos labeled cells in dStr and NACc in pubertal animals compared to adults. The stronger expression in NACc for mid-pubertal rats can be potentially explained by an augmented sensitivity to SCM, leading to higher valuation of the conditioned cue and an enhanced neuronal responding to its presentation. As discussed above, mid-pubertal rats also showed the highest consummatory and motivational behavior for SCM. Although NACs has been considered to be an important brain structure in mediating hedonic evaluation of rewards (Pecina and Berridge, 2000), we could not detect differences in c-fos expression between pubertal and adult rats for this specific region. Since in our experimental setup c-fos expression was examined after presentation of the associated odor cue and not after direct SCM presentation, this might have contributed to a less enhanced neuronal activation of the NACs. Indeed, some studies are indicating a more pronounced role of the NACc in the formation of associations of conditioned and unconditioned stimuli and cue elicited drug seeking (Parkinson et al., 1999; Miller and Marshall, 2005; Chaudhri et al., 2010).

Taken together, the present findings strongly support the importance of striatal structures in the processing of cues related to pleasant stimuli. Furthermore, the enhanced cue-induced c-fos expression in pubertal rats compared to adults indicates an increased neuronal responsiveness toward appetitive stimuli during pubertal development.

\section{CONCLUSIONS}

With the present study we observed higher consummatory behavior and enhanced motivational incentive for a palatable food reward in pubertal rats compared to adult animals. Interestingly, this increased reward sensitivity was most pronounced around mid-puberty (pd 50). The behavioral findings are supported by the measurement of c-fos staining in reward-related brain structures revealing an intensified neuronal response after reward-cue stimulation, specifically for pubertal animals. These findings are demonstrating on the behavioral and also the molecular level that the attributed incentive value of a natural reward is highly increased during adolescence, and this high reward sensitivity seems to be specifically intensified around mid-puberty. In particular, the data on c-fos expression clearly indicate an increased neuronal activity upon reward-cue presentation. It is conceivable that adolescent-typical behavioral patterns such as hyperactivity, risk taking behavior and impulsivity might be potential confounding variables on behavioral performance. However, these adolescent behavioral characteristics appear not to influence the neuronal measures since c-fos expression in sham-trained rats did not differ between pubertal and adult animals.

These findings might be of some translational value for a better understanding of the high vulnerability of teenagers for the initiation of experimental drug use that might precipitate later abuse and dependence. Since natural and drug reinforcers are thought to share common reward pathways (Berridge and Robinson, 1998; Spanagel and Weiss, 1999), the transient enhancement of sensitivity 
and vulnerability toward the rewarding properties of incentives during adolescence might relate as well to the rewarding properties of drugs of abuse, and therefore enhance the attractiveness of these substances for teenagers.

\section{REFERENCES}

Andersen, S. L. (2002). Changes in the second messenger cyclic AMP during development may underlie motoric symptoms in attention deficit/hyperactivity disorder (ADHD). Behav. Brain Res. 130, 197-201.

Andersen, S. L., and Teicher, M.H. (2000). Sex differences in dopamine receptors and their relevance to ADHD. Neurosci. Biobehav. Rev. 24, 137-141.

Anthony, J. C., and Petronis, K. R. (1995). Early-onset drug use and risk of later drug problems. Drug Alcohol Depend. 40, 9-15.

Berridge, K. C., and Robinson, T. E. (1998). What is the role of dopamine in reward: hedonic impact, reward learning, or incentive salience? Brain Res. Brain Res. Rev. 28, 309-369.

Brenhouse, H. C., and Andersen, S. L. (2008). Delayed extinction and stronger reinstatement of cocaine conditioned place preference in adolescent rats, compared to adults. Behav. Neurosci. 122, 460-465.

Casey, B. J., Getz, S., and Galvan, A. (2008). The adolescent brain. Dev. Rev. 28, 62-77.

Chambers, R.A., Taylor, J. R., and Potenza, M. N. (2003). Developmental neurocircuitry of motivation in adolescence: a critical period of addiction vulnerability. Am. J. Psychiatry 160, 1041-1052.

Chaudhri, N., Sahuque, L. L., Schairer, W. W., and Janak, P. H. (2010). Separable roles of the nucleus accumbens core and shell in context- and cue-induced alcohol-seeking. Neuropsychopharmacology 35, 783-791.

Crews, F., He, J., and Hodge, C. (2007). Adolescent cortical development: a critical period of vulnerability for addiction. Pharmacol. Biochem. Behav. 86, 189-199.

Dalley, J. W., Fryer, T. D., Brichard, L., Robinson, E. S., Theobald, D. E., Laane, K., Pena, Y., Murphy, E. R., Shah, Y., Probst, K., Abakumova, I., Aigbirhio, F. I., Richards, H. K., Hong, Y., Baron, J. C., Everitt, B. J., and Robbins, T. W. (2007). Nucleus accumbens D2/3 receptors predict trait impulsivity and cocaine reinforcement. Science 315, 1267-1270.

De Bellis, M. D., Keshavan, M. S., Beers, S. R., Hall, J., Frustaci, K., Masalehdan, A., Noll, J., and Boring, A. M. (2001). Sex differences in brain maturation during childhood and adolescence. Cereb. Cortex 11, 552-557.
Delgado, M. R., Locke, H. M., Stenger, V. A., and Fiez, J. A. (2003). Dorsal striatum responses to reward and punishment: effects of valence and magnitude manipulations. Cogn. Affect. Behav. Neurosci. 3, 27-38.

Desor, J.A., and Beauchamp, G. K. (1987). Longitudinal changes in sweet preferences in humans. Physiol. Behav. 39, 639-641.

Doremus-Fitzwater, T. L., Varlinskaya, E. I., and Spear, L. P. (2010). Motivational systems in adolescence: possible implications for age differences in substance abuse and other risk-taking behaviors. Brain Cogn. 72, 114-123.

Douglas, L.A., Varlinskaya, E. I., and Spear, L.P. (2003). Novel-object place conditioning in adolescent and adult male and female rats: effects of social isolation. Physiol. Behav. 80, 317-325.

Douglas, L.A., Varlinskaya, E. I., and Spear, L. P. (2004). Rewarding properties of social interactions in adolescent and adult male and female rats: impact of social versus isolate housing of subjects and partners. Dev. Psychobiol. 45, 153-162.

Ellgren, M., Artmann, A., Tkalych, O., Gupta, A., Hansen, H. S., Hansen, S. H., Devi, L. A., and Hurd, Y. L. (2008). Dynamic changes of the endogenous cannabinoid and opioid mesocorticolimbic systems during adolescence: THC effects. Eur. Neuropsychopharmacol. 18, 826-834.

Ernst,M., Nelson,E.E., Jazbec, S., McClure, E. B., Monk, C. S., Leibenluft, E., Blair, J., and Pine, D. S. (2005). Amygdala and nucleus accumbens in responses to receipt and omission of gains in adults and adolescents. Neuroimage 25, 1279-1291.

Ernst, M., Romeo, R. D., and Andersen, S. L. (2009). Neurobiology of the development of motivated behaviors in adolescence: a window into a neural systems model. Pharmacol. Biochem. Behav. 93, 199-211.

Galvan, A., Hare, T. A., Parra, C. E., Penn, J., Voss, H., Glover, G., and Casey, B. J. (2006). Earlier development of the accumbens relative to orbitofrontal cortex might underlie risk-taking behavior in adolescents. J. Neurosci. 26, 6885-6892.

Geier, C., and Luna, B. (2009). The maturation of incentive processing and cognitive control. Pharmacol. Biochem. Behav. 93, 212-221.

Gilbert, R. M., and Sherman, I. P. (1970). Palatability-induced polydipsia:

\section{ACKNOWLEDGMENTS}

This study was supported by grants from Land BW (NAR) and DFG (SCHN 958/3-1). We are very thankful to Elisabeth Roebel for excellent technical support.

saccharin, sucrose, and water intake in rats, with and without food deprivation. Psychol. Rep. 27, 319-325.

Grill, H. J., and Norgren, R. (1978). The taste reactivity test. I. Mimetic responses to gustatory stimuli in neurologically normal rats. Brain Res. 143 263-279.

Grippo, A. J., Na, E. S., Johnson, R. F., Beltz, T. G., and Johnson, A. K. (2004). Sucrose ingestion elicits reduced Fos expression in the nucleus accumbens of anhedonic rats. Brain Res. 1019 , 259-264.

Haycock, J. W., Becker, L., Ang, L., Furukawa, Y., Hornykiewicz, O., and Kish, S. J. (2003). Marked disparity between age-related changes in dopamine and other presynaptic dopaminergic markers in human striatum. J. Neurochem. 87, 574-585.

Hodos, W., and Kalman, G. (1963). Effects of increment size and reinforcer volume on progressive ratio performance. J. Exp. Anal. Behav. 6, 387-392.

Hughes, P., and Dragunow, M. (1995). Induction of immediate-early genes and the control of neurotransmitterregulated gene expression within the nervous system. Pharmacol. Rev. 47, 133-178.

Kelley, A. E., and Berridge, K. C. (2002) The neuroscience of natural rewards: relevance to addictive drugs. $J$. Neurosci. 22, 3306-3311.

Knutson, B., Fong, G. W., Adams, C. M. Varner, J. L., and Hommer, D. (2001). Dissociation of reward anticipation and outcome with event-related fMRI. Neuroreport 12, 3683-3687.

Korenbrot, C. C., Huhtaniemi, I. T., and Weiner, R. I. (1977). Preputial separation as an external sign of pubertal development in the male rat. Biol. Reprod. 17, 298-303.

Kringelbach, M. L., and Berridge, K. C. (2009). Towards a functional neuroanatomy of pleasure and happiness. Trends Cogn. Sci. 13, 479-487.

Kuhn, C., Johnson, M., Thomae,A.,Luo, B. Simon, S.A., Zhou, G., and Walker, Q. D. (2009). The emergence of gonadal hormone influences on dopaminergic function during puberty. Horm. Behav. 58, 122-137.

Laviola, G., Macri, S., Morley-Fletcher, S. and Adriani, W. (2003). Risk-taking behavior in adolescent mice: psychobiological determinants and early epigenetic influence. Neurosci. Biobehav. Rev. 27, 19-31.
Macri, S., Adriani, W., Chiarotti, F., and Laviola, G. (2002). Risk taking during exploration of a plus-maze is greater in adolescent than in juvenile or adult mice. Anim. Behav. 64, 541-546.

Miller, C. A., and Marshall, J. F. (2005). Altered Fos expression in neural pathways underlying cue-elicited drug seeking in the rat. Eur. J. Neurosci. 21, 1385-1393.

Mungarndee, S. S., Lundy, R. F. Jr., and Norgren, R. (2008). Expression of Fos during sham sucrose intake in rats with central gustatory lesions. Am. J. Physiol. Regul. Integr. Comp. Physiol. 295, R751-R763.

Nakano, M., and Mizuno, T. (1996). Agerelated changes in the metabolism of neurotransmitters in rat striatum: a microdialysis study. Mech. Ageing Dev. 86, 95-104.

Nance, D. M. (1983). The developmental and neural determinants of the effects of estrogen on feeding behavior in the rat: a theoretical perspective. Neurosci. Biobehav. Rev. 7, 189-211.

Norgren, R., Hajnal,A., and Mungarndee, S.S. (2006). Gustatory reward and the nucleus accumbens. Physiol. Behav. 89, 531-535.

O'Doherty, J.P., Deichmann, R., Critchley, H. D., and Dolan, R. J. (2002). Neural responses during anticipation of a primary taste reward. Neuron 33, 815-826.

Panagis, G., Nomikos, G. G., Miliaressis, E., Chergui, K., Kastellakis, A., Svensson, T. H., and Spyraki, C. (1997). Ventral pallidum self-stimulation induces stimulus dependent increase in c-fos expression in reward-related brain regions. Neuroscience 77, 175-186.

Parkinson, J. A., Olmstead, M. C., Burns, L. H., Robbins, T. W., and Everitt, B. J. (1999). Dissociation in effects of lesions of the nucleus accumbens core and shell on appetitive pavlovian approach behavior and the potentiation of conditioned reinforcement and locomotor activity by D-amphetamine. J. Neurosci. 19, 2401-2411.

Patton, G. C., McMorris, B. J. Toumbourou, J. W., Hemphill, S. A., Donath, S., and Catalano, R. F. (2004). Puberty and the onset of substance use and abuse. Pediatrics 114, e300-e306.

Paus, T., Keshavan, M., and Giedd, J. N. (2008). Why do many psychiatric disorders emerge during adolescence? Nat. Rev. Neurosci. 9, 947-957. 
Paxinos, G., and Watson, C. (1998). The Rat Brain in Stereotaxic Coordinates. San Diego: Academic Press.

Pecina, S., and Berridge, K. C. (2000). Opioid site in nucleus accumbens shell mediates eating and hedonic 'liking' for food: map based on microinjection Fos plumes. Brain Res. 863, 71-86.

Piazza, P. V., Deminiere, J. M., Le, M. M., and Simon, H. (1989). Factors that predict individual vulnerability to amphetamine self-administration. Science 245, 1511-1513.

Post, G. B., and Kemper, H. C. (1993). Nutrient intake and biological maturationduringadolescence. TheAmsterdam growth and health longitudinal study. Eur. J. Clin. Nutr. 47, 400-408.

Powell, K. (2006). Neurodevelopment: how does the teenage brain work? Nature 442, 865-867.

Rodriguez de Fonseca, F., Ramos, J. A., Bonnin, A., and Fernandez-Ruiz, J. J. (1993). Presence of cannabinoid binding sites in the brain from early postnatal ages. Neuroreport 4, 135-138.

Sanchis-Segura, C., and Spanagel, R. (2006). Behavioural assessment of drug reinforcement and addictive features in rodents: an overview. Addict. Biol. 11, 2-38.

Schneider, M. (2008). Puberty as a highly vulnerable developmental period for the consequences of cannabis exposure. Addict. Biol. 13, 253-263.

Schneider, M., Heise, V., and Spanagel, R. (2010). Differential involvement of the opioid receptor antagonist naloxone in motivational and hedonic aspects of reward. Behav. Brain Res. 208, 466-472.

Schneider, M., and Spanagel, R. (2008). Appetitive odor-cue conditioning attenuates the acoustic startle response in rats. Behav. Brain Res. 189, 226-230.

Shram, M. J., Funk, D., Li, Z., and Le, A. D. (2008). Nicotine self-administration, extinction responding and reinstatement in adolescent and adult male rats: evidence against a biological vulnerability to nicotine addiction during adolescence. Neuropsychopharmacology 33, 739-748.

Sisk, C. L., and Foster, D. L. (2004). The neural basis of puberty and adolescence. Nat. Neurosci. 7, 1040-1047.

Solinas, M., and Goldberg, S. R. (2005). Motivational effects of cannabinoids and opioids on food reinforcement depend on simultaneous activation of cannabinoid and opioid systems. Neuropsychopharmacology 30, 2035-2045.

Solinas, M., Yasar, S., and Goldberg, S. R. (2007). Endocannabinoid system involvement in brain reward processes related to drug abuse. Pharmacol. Res. 56, 393-405.

Spanagel, R. (2009). Alcoholism: a systems approach from molecular physiology to addictive behavior. Physiol. Rev. 89, 649-705.

Spanagel, R., and Weiss, F. (1999). The dopamine hypothesis of reward: past and current status. Trends Neurosci. 22, 521-527.

Spear, L. P. (2000). The adolescent brain and age-related behavioral manifestations. Neurosci. Biobehav. Rev. 24, 417-463.

Spear, L. P., and Varlinskaya, E. I. (2010). Sensitivity to ethanol and other hedonic stimuli in an animal model of adolescence: Implications for prevention science? Dev. Psychobiol. 52, 236-243.
Stafford, D., LeSage, M. G., and Glowa, J. R. (1998). Progressive-ratio schedules of drug delivery in the analysis of drug self-administration: a review. Psychopharmacology (Berl.) 139, 169-184.

Streefland, C., Farkas, E., Maes, F. W., and Bohus, B. (1996). C-fos expression in the brainstem after voluntary ingestion of sucrose in the rat. Neurobiology (Bp) 4, 85-102.

Tarazi, F. I., and Baldessarini, R. J. (2000) Comparative postnatal development of dopamine $\mathrm{D}(1), \mathrm{D}(2)$ and $\mathrm{D}(4)$ receptors in rat forebrain. Int. J. Dev. Neurosci. 18, 29-37.

Vaidya, J. G., Grippo, A. J., Johnson, A. K. and Watson, D. (2004). A comparative developmental study of impulsivity in rats and humans: the role of reward sensitivity. Ann. N. Y. Acad. Sci. 1021, 395-398.

Varlinskaya, E. I., and Spear, L. P. (2008). Social facilitation induced by pharmacological activation of mu opioid receptors: impact of age, sex and stress. Poster presented at the Society for Neuroscience meeting 2008, Washington, DC. Ref Type: Conference Proceeding.

Wenger, T., Gerendai, I., Fezza, F., Gonzalez, S., Bisogno, T., Fernandez-Ruiz, J., and Di Marzo, V. (2002). The hypothalamic levels of the endocannabinoid, anandamide, peak immediately before the onset of puberty in female rats. Life Sci. 70, 1407-1414.

Wills, T. A., Vaccaro, D., and McNamara, G. (1994). Novelty seeking, risk taking, and related constructs as predictors of adolescent substance use: an application of Cloninger's theory. J. Subst. Abuse 6, 1-20.
Wilmouth, C. E., and Spear, L. P. (2009). Hedonic sensitivity in adolescent and adult rats: taste reactivity and voluntary sucrose consumption. Pharmacol. Biochem. Behav. 92, 566-573.

Wise, R. A. (2004). Dopamine, learning and motivation. Nat. Rev. Neurosci. 5, 483-494.

Wise, R. A. (2009). Roles for nigrostriatalnot just mesocorticolimbic-dopamine in reward and addiction. Trends Neurosci. 32, 517-524.

Zhang, F., Zhou, W., Liu, H., Zhu, H., Tang, S., Lai, M., and Yang, G. (2005). Increased c-Fos expression in the medial part of the lateral habenula during cue-evoked heroin-seeking in rats. Neurosci. Lett. 386, 133-137.

Conflict of Interest Statement: The authors declare that the research was conducted in the absence of any commercial or financial relationships that could be construed as a potential conflict of interest.

Received: 05 May 2010; paper pending published: 02 June 2010; accepted: 18 June 2010; published online: 13 July 2010.

Citation: Friemel CM, Spanagel $R$ and Schneider M (2010) Reward sensitivity for a palatable food reward peaks during pubertal developmental in rats. Front. Behav. Neurosci. 4:39. doi: 10.3389/ fnbeh.2010.00039

Copyright (c) 2010 Friemel, Spanagel and Schneider. This is an open-access article subject to an exclusive license agreement between the authors and the Frontiers Research Foundation, which permits unrestricted use, distribution, and reproduction in any medium, provided the original authors and source are credited. 